\title{
El intercambio virtual: experiencias desde instituciones en el Caribe colombiano
}

\author{
The virtual exchange: experiences from institutions in the Colombian Caribbean \\ Tania Isabel Lafont Castillo1; ori@pca.edu.co \\ Luisa Fernanda Echeverría King²; lecheverriaking@gmail.com \\ Liliana Patricia Álvarez Ruíz³; liliana.alvarez@cecar.edu.co
}

\begin{abstract}
Resumen
El intercambio virtual busca generar espacios de interacción cultural, de investigación, de idiomas, entre otras modalidades entre grupos de estudiantes y docentes ubicados en diversas localidades o países. En la educación superior se usa entre instituciones como una estrategia para el desarrollo de competencias en estudiantes, así como para la construcción de comunidades de aprendizaje. Con el fin de caracterizar las acciones de intercambio virtual para estudiantes realizadas en las instituciones adscritas al Nodo Caribe de la Red Colombiana para la Internacionalización de la Educación Superior $(\mathrm{RCl})$, se realizó un estudio cuantitativo descriptivo, donde se encuestaron a las 26 instituciones miembro, de las cuales 24 realizan acciones de intercambio virtual. Resaltan las modalidades como el intercambio académico, pasantías de investigación, en empresas y esquemas de aprendizaje colaborativo en línea. Los países principales con los que se realizan estas actividades son México, Argentina y Perú. Existen desafíos para su implementación especialmente por la capacitación de docentes, seguimiento y monitoreo a estudiantes y sus resultados de aprendizaje. Es necesario contar con una buena infraestructura, conectividad y docentes capacitados en aspectos técnicos y pedagógicos para facilitar el éxito en la ejecución de estos intercambios, así como generar procedimientos institucionales que faciliten su implementación.
\end{abstract}

Palabras clave: intercambio virtual, internacionalización, educación superior, innovación educativa.

\section{Abstract}

The virtual exchange seeks to create spaces for cultural, research, and language interactions, among others, between groups of students and teachers based in different countries. In higher education, it is used as an institutional strategy to provide students with competencies and create learning communities. In order to characterize the student's virtual exchange methods carried out within the higher education institutions attached to the Caribbean Node of the Colombian Network for Internationalization of Higher Education (RCl), a descriptive quantitative study was conducted, where 26 member institutions were surveyed, of which 24 carry out student's virtual exchange actions. Virtual exchange types for students such as academic exchange, internships for research purposes, internships in companies, and collaborative online learning schemes stand out. The main country partners for these activities are Mexico, Argentina, and Peru. There are implementation challenges such as teacher training and student's monitoring and reaching of learning outcomes. Also, a good infrastructure, connectivity, skilled teachers in technical and pedagogical aspects and the creation of institutional procedures for these interactions play an important role in the success of the virtual exchange.

Keywords: virtual exchange, internationalization, higher education, educational innovation.

\footnotetext{
${ }^{1}$ Corporación Politécnico de la Costa Atlántica (Colombia)

${ }^{2}$ Servicio Nacional de Aprendizaje [SENA] (Colombia).

${ }^{3}$ Corporación Universitaria del Caribe [CECAR] (Colombia).
} 


\section{INTRODUCCIÓN}

El intercambio virtual es un concepto del siglo XXI, fruto de la sociedad del conocimiento y el boom de las tecnologías de la información y las comunicaciones (TIC). Las TIC han revolucionado la forma como se facilita el acceso a conocimiento, masificando las experiencias formativas y democratizando oportunidades de intercambio de conocimientos. La movilidad, en especial en el marco de procesos formativos se ha evidenciado como una experiencia elitista y excluyente por racionalidad económica (Camacho Lizárraga, 2017), por lo cual cada vez más instituciones se encuentran ejecutando actividades de intercambio virtual con cooperantes internacionales, que impulsen no sólo la inclusión de las dimensiones internacionales e interculturales en la formación, sino también en las demás funciones misionales. Verzella (2018) resalta igualmente la importancia de los intercambios virtuales para el aprendizaje de competencias asociadas a la ciudadanía global, la conciencia intercultural y la mentalidad abierta cosmopolita en tiempos caracterizados por un resurgimiento del nacionalismo.

La Red Colombiana para la Internacionalización de la Educación Superior ( $\mathrm{RCl})$, alianza entre instituciones de educación superior (IES) para el fomento de la internacionalización, la visibilidad y el trabajo en red con instituciones y actores del entorno. La $\mathrm{RCl}$ se compone de nueve nodos regionales (Orinoquía, Bogotá, Caribe, Occidente, Cafetero, Sur Occidente, Centro, Oriente y Tolima Grande). El Nodo Caribe cuenta con 26 instituciones miembro, ubicadas en los siete departamentos de la costa Atlántica colombiana. Se presentan en este artículo los resultados de una investigación que busca caracterizar la implementación de esquemas de intercambio virtual para estudiantes realizadas por instituciones adscritas al Nodo Caribe de la Red Colombiana para la Internacionalización en el mes de octubre de 2020.

\section{MARCO TEÓRICO}

\subsection{EL CONCEPTO DEL INTERCAMBIO VIRTUAL}

El intercambio virtual es una expresión de la sociedad del conocimiento, impactada por avalanchas de información y marcada por las tecnologías de información. Los intercambios virtuales han ganado relevancia a través de los años, y recientemente, como respuesta a la pandemia del Covid-19, aunque se considera que el intercambio virtual sentó sus bases a finales de los años 80 (Stevens Initiative, 2019). Los intercambios virtuales pueden ser vistos como experiencias de enseñanza-aprendizaje innovadoras. Para esto, se deben cumplir ciertos requisitos. El rol del profesor en el marco de los intercambios virtuales es marcado y juega una función como guía o acompañante en el proceso. El estudiante, asimismo, se convierte en un constructor de su proceso de aprendizaje. Galvan Acosta y Moreno Correa (2017) indican que las experiencias de intercambio virtual son significativas en el marco de la sociedad del conocimiento, dado que suponen una oportunidad de vivir una aproximación con personas de otras latitudes, costumbres y entendimientos diversos de la realidad. Sin embargo, a pesar de su importancia existe poca claridad sobre el concepto de intercambio virtual como evidencian los autores Jager et al. (2019) en el estudio titulado: "Virtual Exchange as Innovative Practice across Europe Awareness and Use in Higher Education", producto del Proyecto EVOLVE, el cual tuvo como objetivo incorporar el intercambio virtual como una forma innovadora de aprendizaje 
internacional colaborativo en todas las disciplinas en las IES de Europa. Los autores hallaron que el concepto de intercambio virtual se confunde con la educación en línea y la educación virtual. También encontraron que las disciplinas que más reportaron el desarrollo de intercambio virtual fueron: arte y humanidades, educación, ciencias sociales y periodismo y que existe un bajo grado de integración del intercambio virtual en el currículo de las IES objeto de estudio.

Por otro lado, existen diversos tipos de intercambio virtual concebidos como experiencias formativas con el apoyo de la tecnología y que involucran personas de diversos orígenes culturales que son geográficamente distantes, construyendo de esta forma aprendizaje significativo con el apoyo de educadores. O'Dowd (2018) señala los siguientes tipos: el teletandem o telecolaboración para el fomento del aprendizaje de lenguas extranjeras; los intercambios virtuales específicos de la asignatura o equipo virtual global para el fomento del aprendizaje de habilidades interculturales necesarias para el desempeño en el sector productivo; el aprendizaje internacional en línea que implica el diseño de un contenido compartido, acordado entre diferentes universidades para desarrollar competencias interculturales; proveedor de servicios que implican iniciativas promovidas por diferentes organizaciones que participan en el desarrollo del plan de estudios y el entorno virtual. El teletandem ha gozado de mucha importancia para el aprendizaje e intercambio en lenguas extranjeras. Telles (2015) explica que el teletandem es un modo de telecolaboración virtual autónoma para el aprendizaje de lenguas extranjeras en el que dos estudiantes se ayudan mutuamente a aprender su propio idioma. En todo caso y dependiendo de la modalidad, se fomentará el aprendizaje individual o en equipo.

\subsection{BENEFICIOS DEL INTERCAMBIO VIRTUAL}

Mucho se ha discutido sobre los beneficios del intercambio virtual, especialmente cuando este se gestiona con cooperantes internacionales. Entre los beneficios más importantes, se observa un aprendizaje auto dirigido, la construcción de comunidades de aprendizaje internacionales y el desarrollo de habilidades blandas como el trabajo en equipo, liderazgo, la autogestión e incluso el desarrollo de competencias en idiomas extranjeros, en el caso de que estos intercambios se realicen con instituciones de países no hispanoparlantes. Guth et al. (2014) exponen que, a la hora de organizar intercambios virtuales, se le da prioridad al desarrollo de competencias interculturales, fomentar aprendizaje de lenguas extranjeras, apoyar el desarrollo de competencias TIC y autonomía de los procesos enseñanza-aprendizaje.

En este sentido el intercambio virtual, al ser realizado con entidades extranjeras, apoya el aprendizaje y el intercambio intercultural y ofrece la oportunidad para que los estudiantes puedan formar parte de comunidades de aprendizaje virtuales (Schenker, 2013). También, siguiendo a esta autora, el intercambio virtual genera oportunidades para aprendizaje significativo por medio de comunicaciones auténticas, favoreciendo, en el caso del aprendizaje de lenguas extranjeras, una motivación para los estudiantes frente al desarrollo de competencias en la cultura y lengua destino. En contraste, los autores Rienties et al. (2020) en su estudio de corte longitudinal titulado: "The impact of virtual exchange on TPACK and foreign language competence: reviewing a large-scale implementation across 23 virtual exchanges", cuyo objetivo fue indagar en qué medida el desarrollo de intercambio virtual suscita el incremento de las habilidades tecnológicas, pedagógicas y la competencia lingüística en estudiantes, evidenciaron que la estrategia de intercambio virtual no genera la competencia por ósmosis por lo cual los 
autores recomiendan que para el desarrollo de futuros estudios se considere la preexistencia de estas habilidades en los sujetos de estudio.

\subsection{EVALUACIÓN DEL INTERCAMBIO VIRTUAL}

La evaluación del intercambio virtual se ha evidenciado como un reto para docentes alrededor del mundo. Tiven et al. (2018) exponen que la base para el diseño y ejecución de un buen intercambio virtual son los resultados de aprendizaje, los cuales pueden contar con métodos de evaluación diferentes. En todo caso, las autoras sugieren concentrarse en el desarrollo de los resultados de aprendizaje por parte de los estudiantes y no en una competencia final. También se observa que las instituciones generan rúbricas para evaluar las competencias adquiridas en el marco del intercambio virtual o utilizan algunas diseñadas por asociaciones internacionales de reconocimiento o incluso por otras instituciones. Una de las rúbricas reconocidas internacionalmente es "Value" desarrollada por parte de expertos de la Association of American Colleges and Universities (2009) para medir la competencia y el conocimiento intercultural, esta puede usarse en el marco de intercambios virtuales interculturales.

La autora Deardoff (2015) expone que para evaluar el aprendizaje del estudiante pueden utilizarse métodos directos e indirectos. Los métodos directos son los que evidencian el conocimiento mediante trabajos, proyectos, exámenes o rendimiento estudiantil. Por su parte, los métodos indirectos se basan en la percepción del estudiante entorno a su aprendizaje, algunos ejemplos son: la aplicación de encuestas, entrevistas y grupos focales (Deardoff, 2015). En contraste, para evaluar el aprendizaje de los estudiantes la autora Guadamillas Gómez (2017), recomienda organizar un plan de tareas o entregables asociados al proyecto, los cuales deberían ser trabajados en equipos de estudiantes en el mejor de los casos binacionales. Asimismo, la autora recomienda para efectos de evaluar la satisfacción de los estudiantes frente a la ejecución y resultados del proyecto aplicar cuestionarios a su inicio y cierre.

En todo caso se requiere que toda estrategia de intercambio virtual pueda contar con un plan de monitoreo y evaluación a partir de los objetivos del mismo, el cual sea conocido por todos los socios y participantes en el proceso (The Collaboratory, 2019).

\subsection{DESAFÍOS PARA LA IMPLEMENTACIÓN DEL INTERCAMBIO VIRTUAL}

Entre los desafíos evidenciados frente al intercambio virtual en términos de capacidades institucionales resalta el hecho de que estas actividades no siempre hacen parte de asignaturas concretas, por lo cual no son consideradas en los procedimientos para la evaluación de los cursos. Al respecto, Cavalari (2019) relaciona situaciones pedagógicas e institucionales que no permiten la homologación de estos saberes como la incompatibilidad entre los currículos entre la propia institución y la aliada en el exterior. Asimismo, la autora resalta la existencia de problemas asociados a la infraestructura propia de la institución como computadores obsoletos. Por su parte, los autores Garcés y O'Dowd (2020) exponen la necesidad de reconocimiento institucional. Lo que comprende que los administrativos y directivos reconozcan las potencialidades del intercambio virtual, sino éste se quedará como una simple estrategia micro-curricular.

Por otra parte, los docentes participantes en estas experiencias expresan que planear y ejecutar este tipo de actividades requiere de tiempo y esfuerzo, por lo cual las instituciones deberían tener 
formas para reconocer este tipo de trabajo realizado por los docentes (The Evaluate Group, 2019). Estos factores tensionan el rol del docente en el desarrollo del intercambio virtual y a su vez demandan competencias para formar, guiar, asesorar y evaluar a estudiantes por medio de la virtualidad. Moreira-Segura y Delgadillo-Espinoza (2015) explican que el profesor en el marco de los intercambios virtuales se trata de un guía o facilitador del proceso, sin imponerse o monopolizar conocimiento, promoviendo la participación activa de los involucrados. Asimismo, deben tener organizado el diseño y ejecución del intercambio virtual y a su vez alinear estas planeaciones con las necesidades y requerimientos institucionales (Cavalari y Aranha, 2019). Por lo cual, la capacitación de los docentes es un aspecto central para poder ejecutar acciones de intercambio virtual.

\section{MATERIAL Y MÉTODOS}

\section{Objetivo}

El objetivo de este estudio es caracterizar las acciones de intercambio virtual para estudiantes realizadas en las instituciones adscritas al Nodo Caribe de la Red Colombiana para la Internacionalización de la Educación Superior ( $\mathrm{RCl}$ ).

Del cual se desglosan los siguientes objetivos específicos:

1. Describir la existencia y las tipologías de intercambio virtual estudiantil adelantadas por las IES del Nodo Caribe.

2. Revisar prácticas de evaluación de los intercambios virtuales

3. Relacionar las áreas de conocimiento, niveles de formación y países socios con los que se realizan acciones de intercambio virtual estudiantil

4. Identificar desafíos en la implementación de esquemas de intercambios virtuales estudiantiles

\section{Diseño}

Esta investigación es principalmente de tipo cuantitativa y cuenta con un diseño descriptivo. Según Niño (2011) los estudios descriptivos tienen como propósito "describir la realidad objeto de estudio, un aspecto de ella, sus partes, sus clases, sus categorías o las relaciones que se pueden establecer entre varios objetos, con el fin de esclarecer una verdad, corroborar un enunciado o comprobar una hipótesis" (p. 34). Por otra parte, para profundizar en la caracterización, se incluyen dos preguntas abiertas sobre los desafíos y sugerencias para la implementación de intercambios virtuales, las cuales se analizarán siguiendo el paradigma cualitativo, con categorías y subcategorías.

\section{Participantes}

Esta investigación toma como muestra las instituciones adscritas al Nodo Caribe de la Red Colombiana para la Internacionalización de la Educación Superior (RCl). El Nodo Caribe está conformado por 26 instituciones de educación superior localizadas en diferentes ciudades de la costa caribe (figura 1): 


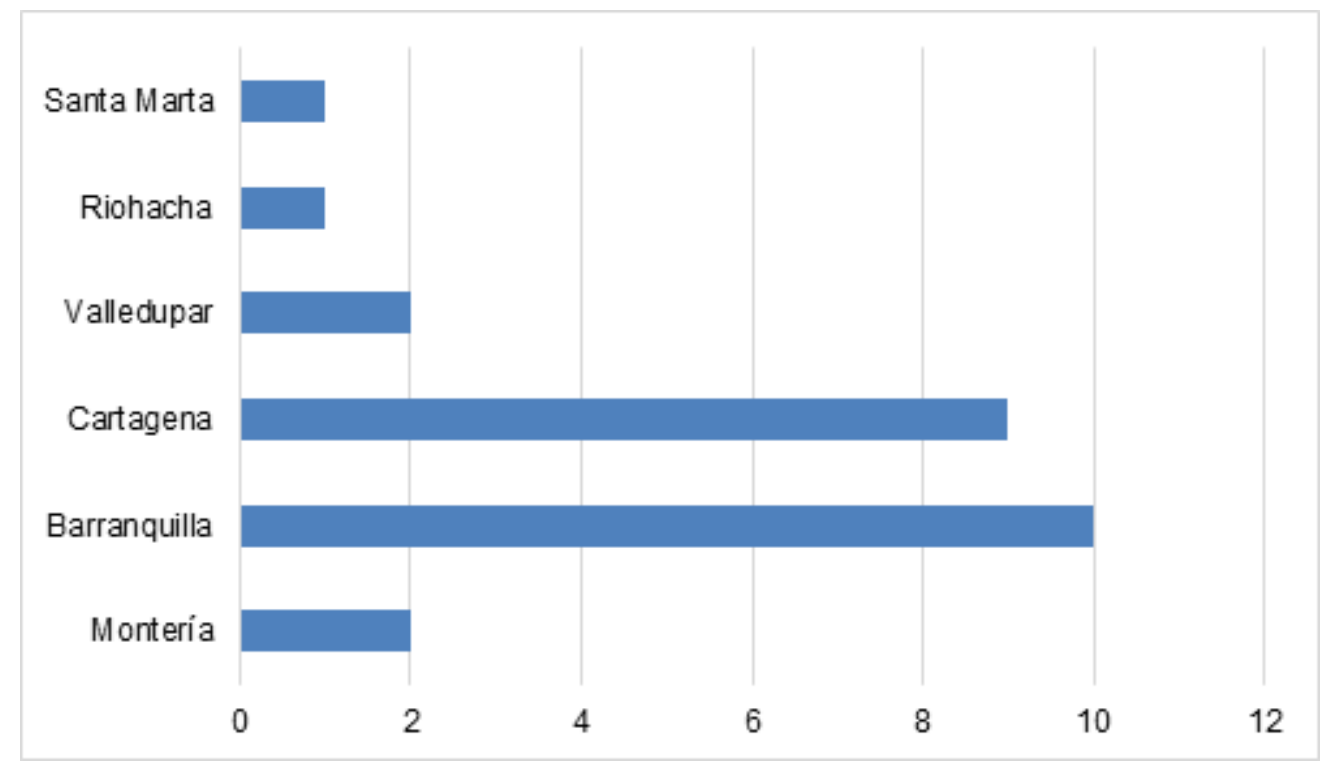

Figura 1. Ciudades de las Instituciones objeto de estudio

En Colombia las IES, según su carácter académico se clasifican en: Instituciones Técnicas Profesionales, Instituciones Tecnológicas, Instituciones Universitarias o Escuelas tecnológicas y Universidades (Congreso de Colombia, 1992). En la encuesta fueron identificados los tipos de IES participantes, el mayor número fueron universidades (17), le siguen Instituciones Universitarias (7) y por último Institución Técnica Profesional y la Institución Tecnológica cada una con (1) como se observa en la figura 2:

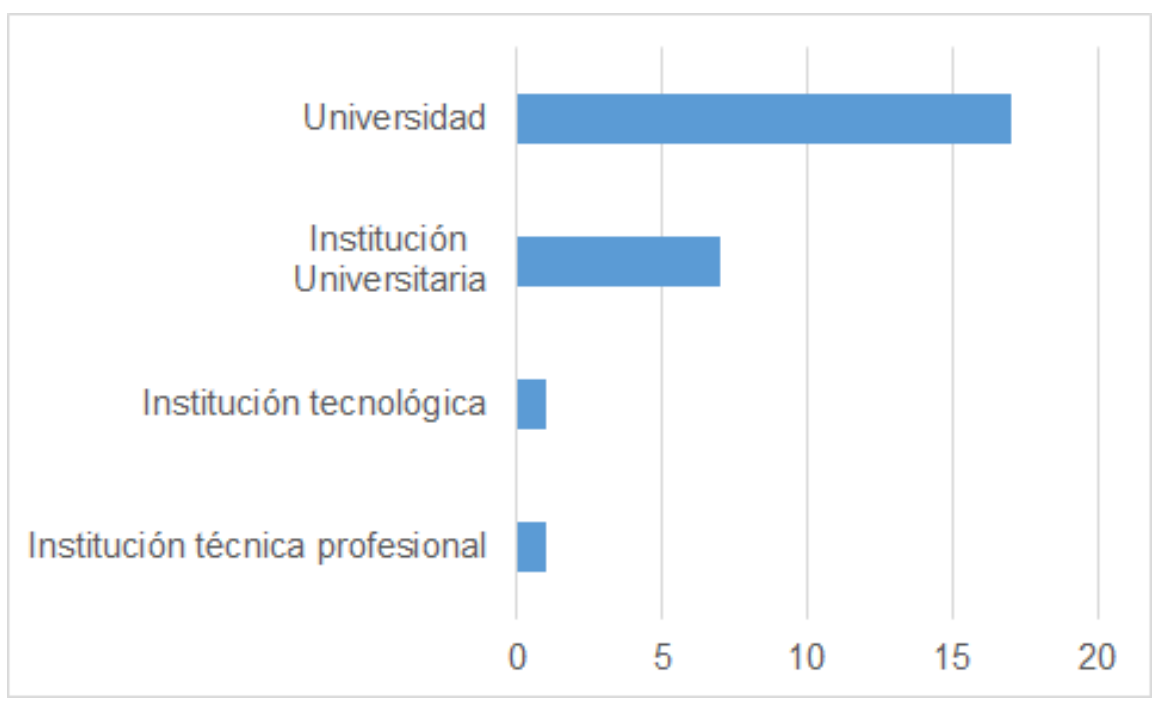

Figura 2. Tipo de Instituciones que participaron en la encuesta

El instrumento fue diligenciado por 26 funcionarios de las IES objeto de estudio, uno por institución. La Figura 3 ilustra que 20 corresponden a Jefes de la Oficina Internacional, mientras que 5 son colaboradores y uno es "coordinador". 


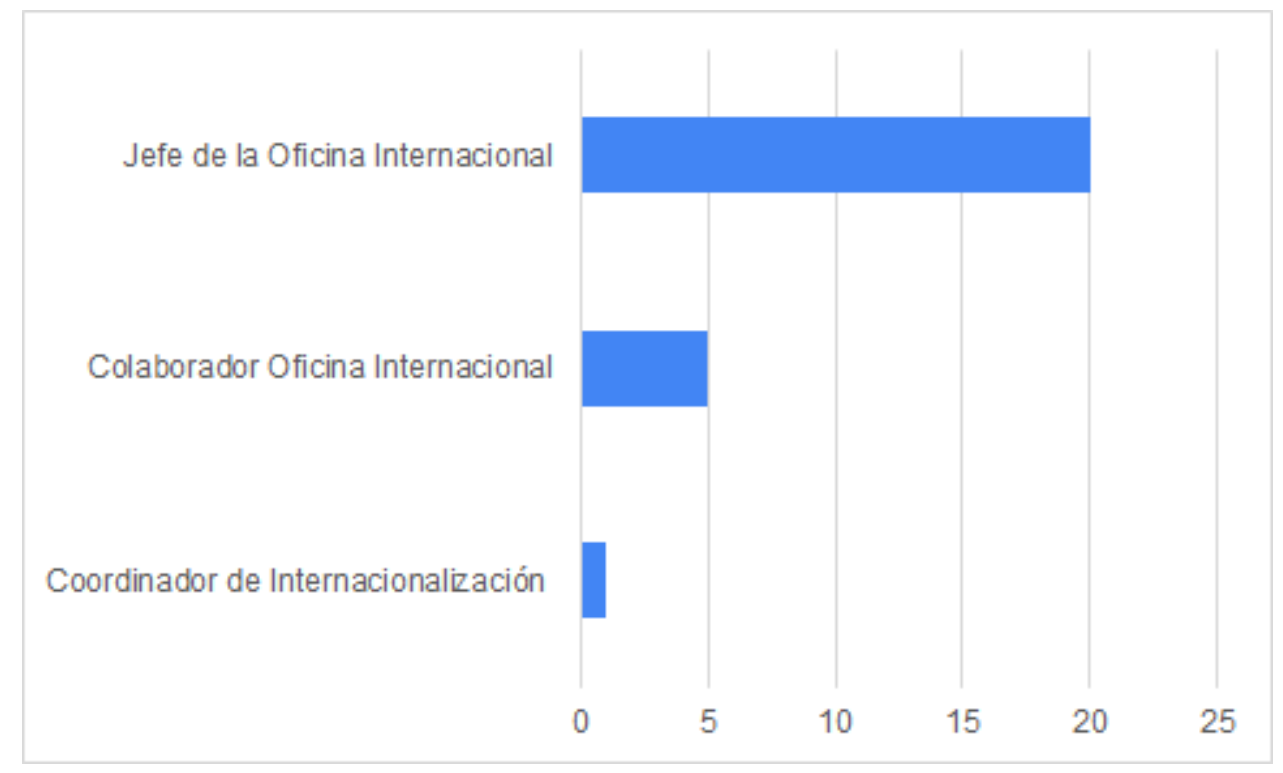

Figura 3. Cargo de quien diligencia la encuesta

\section{Instrumento y procedimiento}

Para la recogida de información se generó un cuestionario mediante Google Forms. Según Behar (2008), "un cuestionario consiste en un conjunto de preguntas respecto a una o más variables a medir. El cuestionario cuenta con dos tipos de preguntas cerradas y abiertas" (p.64).

La encuesta aplicada cuenta con 16 interrogantes y está divida en secciones conforme los objetivos del estudio. La tabla 1 relaciona cada sección con el respaldo teórico:

Tabla 1. Secciones del instrumento y respaldo teórico

\begin{tabular}{|c|l|l|}
\hline \multicolumn{2}{|c|}{ Sección } & \multicolumn{1}{c|}{ Respaldo teórico } \\
\hline $\begin{array}{l}\text { 1. Identificar los tipos de intercambio } \\
\text { virtual estudiantil }\end{array}$ & Revisión de las tipologías propuestas por O’Dowd (2018). \\
\hline 2. Revisar prácticas de evaluación & $\begin{array}{l}\text { Contrastar el uso de métodos directos e indirectos propuestos } \\
\text { por Deardoff (2015) } \\
\text { para la evaluación de los intercambios virtuales. }\end{array}$ \\
\hline $\begin{array}{l}\text { Relacionar áreas de conocimiento, } \\
\text { niveles de formación y socios para el } \\
\text { estudiantil de intercambio virtual }\end{array}$ & $\begin{array}{l}\text { Las preguntas de esta sección fueron adaptadas del estudio de } \\
\text { (Schenker, 2013) conforme a los socios para realizar } \\
\text { intercambio virtual. }\end{array}$ \\
\hline $\begin{array}{l}\text { Identificar desafíos de cara a la la } \\
\text { continuidad del intercambio virtual } \\
\text { estudiantil. }\end{array}$ & $\begin{array}{l}\text { Contrastar los planteamientos de Cavalari (2019); Cavalari y } \\
\text { Aranha (2019); Moreira-Segura y Delgadillo-Espinoza (2015) en } \\
\text { el contexto de las IES del Nodo Caribe }\end{array}$ \\
\hline
\end{tabular}


Las preguntas del cuestionario fueron sometidas a validación por juicio de expertos, quienes brindaron sus observaciones a aspectos relacionados con la redacción y planteamiento de las preguntas. Posterior a la revisión de los expertos las autoras realizaron los ajustes pertinentes y consolidaron la versión final del instrumento, dando un lapso de tiempo del 2 al 16 de octubre del 2020 a las IES objeto de análisis.

Seguidamente se extrajeron las respuestas y fueron tabuladas por medio de la herramienta Excel. Las preguntas abiertas fueron categorizadas según el análisis cualitativo de contenido, creándose redes semánticas por medio del software MAXQDA.

\section{RESULTADOS}

Los resultados de la presente encuesta son presentados de manera agrupada dando respuesta a los objetivos específicos planteados.

\section{Objetivo No 1. Describir la existencia y las tipologías de intercambio virtual estudiantil adelantadas por las IES del Nodo Caribe.}

La primera pregunta indagaba sobre la realización de iniciativas de intercambio virtual para estudiantes, en las IES objeto de estudio. Esta respuesta se encuentra registrada en la Figura 4, indicando que 24 IES realizan acciones de intercambio virtual estudiantil. Una institución no realiza, y otra no sabe. Esto indica que la gran mayoría de las IES del Nodo Caribe colombiano realizan intercambios virtuales.

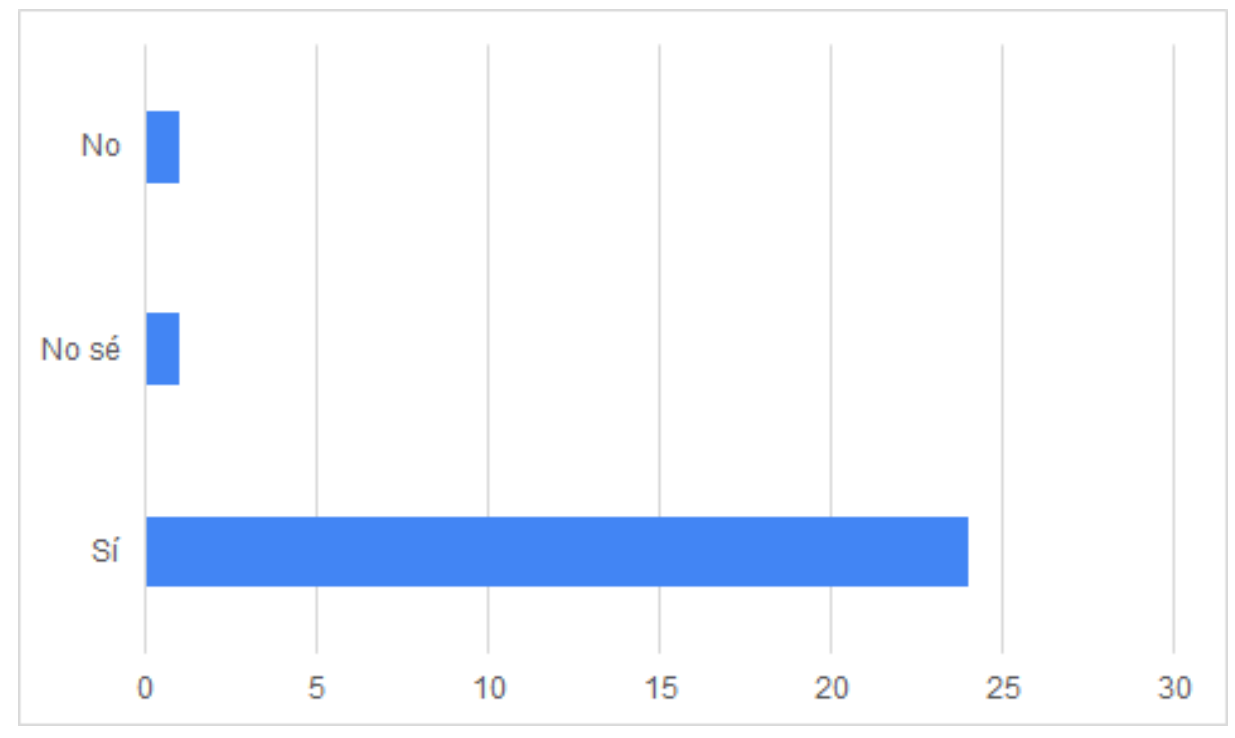

Figura 4. Iniciativas de intercambio virtual

La pregunta sobre tipos de intercambio virtual fue de selección múltiple, permitiendo a los 26 encuestados elegir varias opciones, entre las ocho propuestas. El tipo de actividad más significativa fue el intercambio académico virtual, escogido por 25 IES como la principal actividad de colaboración virtual, representando el $32 \%$ de la muestra, le sigue la pasantía de 
investigación virtual con un 23\%, luego el aprendizaje colaborativo en línea COIL con 16\%, y la práctica empresarial virtual con $14 \%$. Por su parte, las actividades de nivel más bajo de participación en la muestra son: los intercambios lingüísticos con un $9 \%$, las clases espejo con un 4\%; eventos y docentes invitados virtuales cada una representando un $1 \%$, lo anterior se encuentra registrado en la Figura 5.

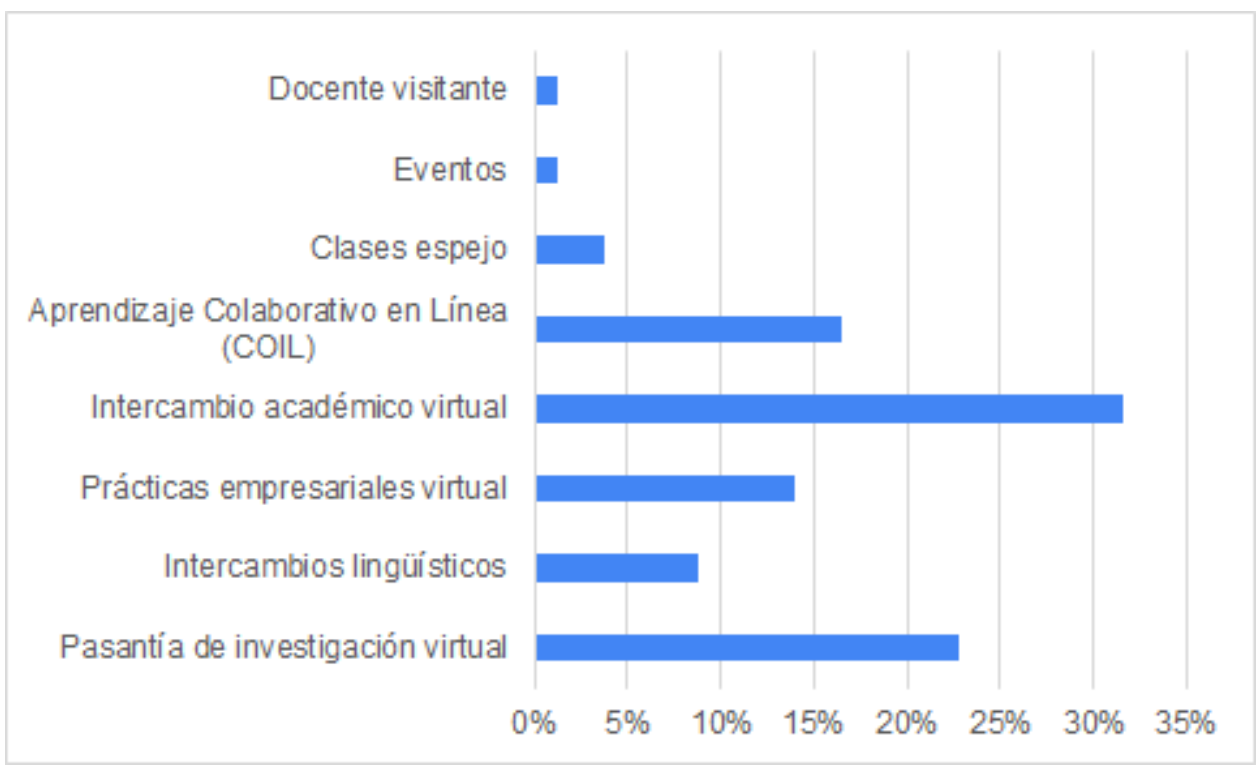

Figura 5. Tipos de intercambio virtual

\section{Objetivo No 2. Revisar prácticas de evaluación de los intercambios virtuales}

Frente a las prácticas de evaluación (figura 6), las instituciones utilizan métodos como el informe escrito con 10 respuestas, el método de evaluación por proyectos y el examen ambas con 8. Siguen 6 IES que evalúan con rúbricas, por último 1 con encuesta y portafolios virtuales. De manera trasversal, se usan resultados de aprendizaje para medir el desempeño de los estudiantes.

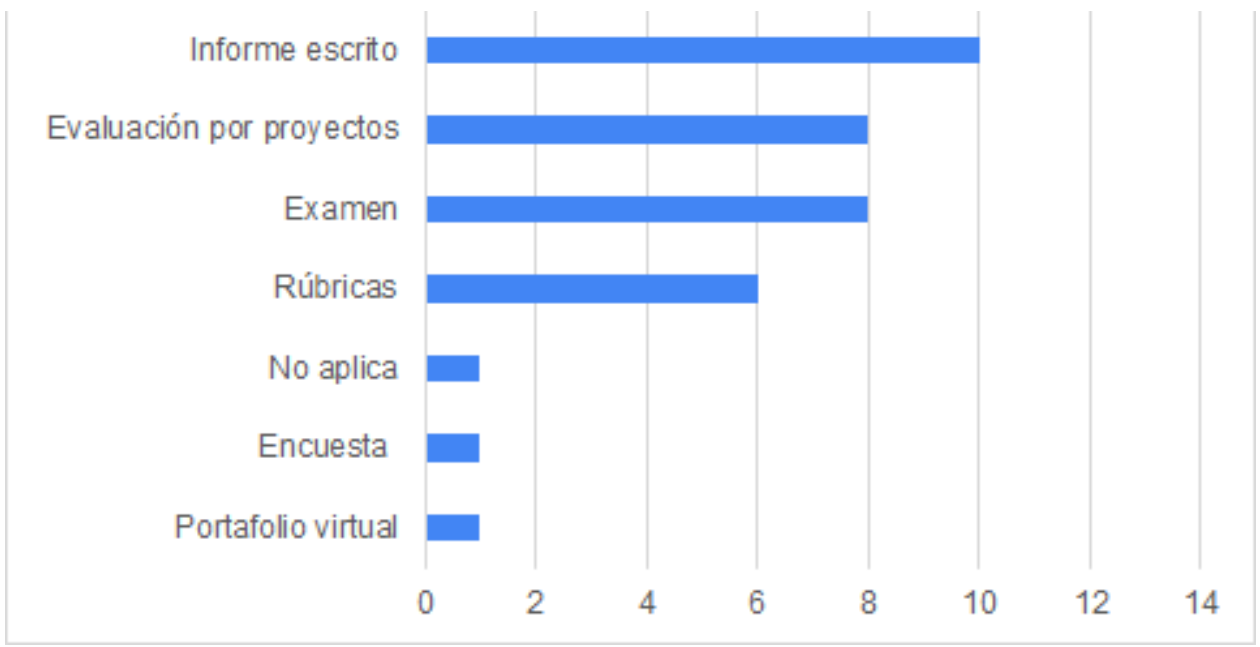

Figura 6. Prácticas de evaluación del intercambio virtual 
A continuación, se preguntó sobre la forma de evaluar la satisfacción de los estudiantes que han participado en procesos de intercambio virtual, aportando varias opciones para elegir (figura 7). 18 IES seleccionaron la encuesta, 11 utilizan la entrevista; le sigue el informe de satisfacción 10 IES; y los dos niveles más bajos fueron el método mixto y no aplica ninguna de las anteriores cada opción con 1 IES.

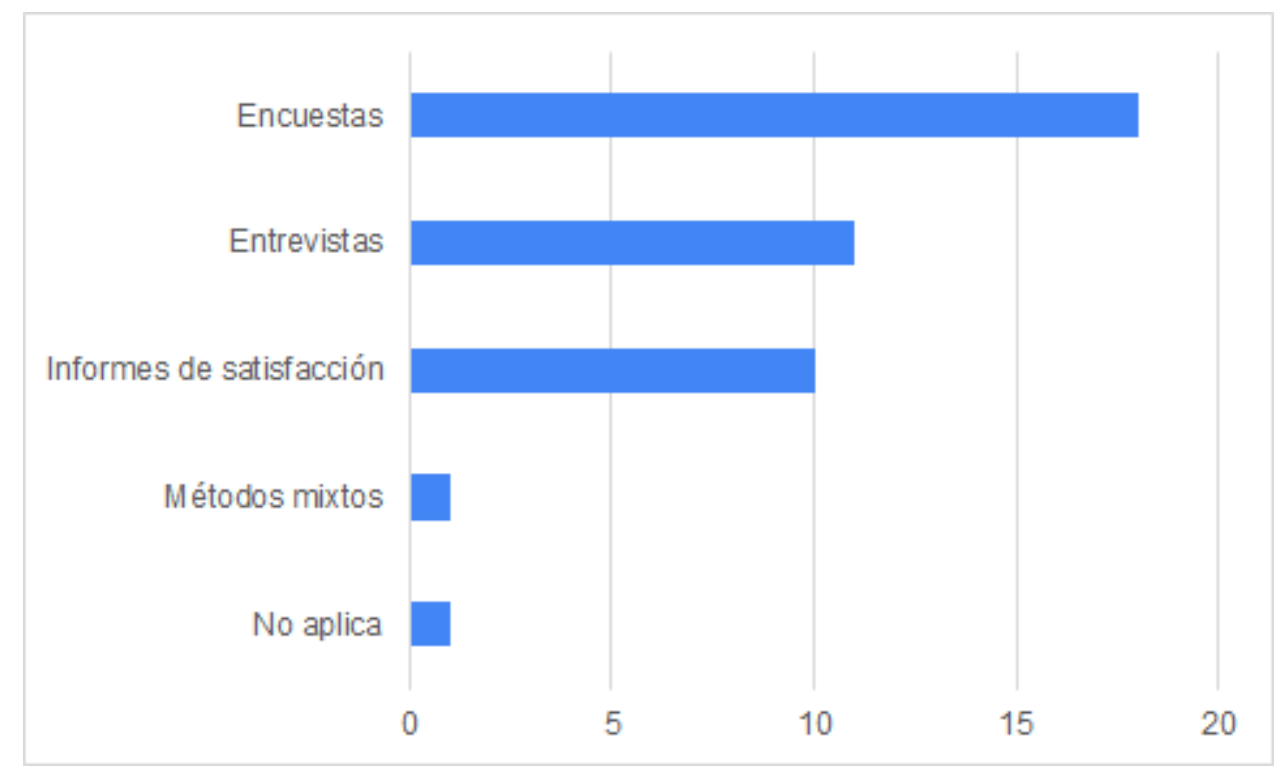

Figura 7. Prácticas de evaluación de la satisfacción estudiantil respecto al intercambio virtual

El estudio también indaga sobre los aspectos que las Instituciones de Educación Superior tienen en cuenta para evaluar la experiencia del intercambio virtual, brindando varias opciones (figura 8). Los hallazgos señalan que 22 IES seleccionaron el desarrollo de competencias interculturales, seguido de los aprendizajes técnicos o disciplinares con 14; el uso de herramientas tecnológicas es escogida por 13, y el aprendizaje de una segunda lengua con 9 IES.

Es interesante encontrar que la competencia intercultural, al ser una competencia blanda, sea el aspecto más importante en las IES, y que incluso tenga un valor mayor que los aprendizajes técnicos. 


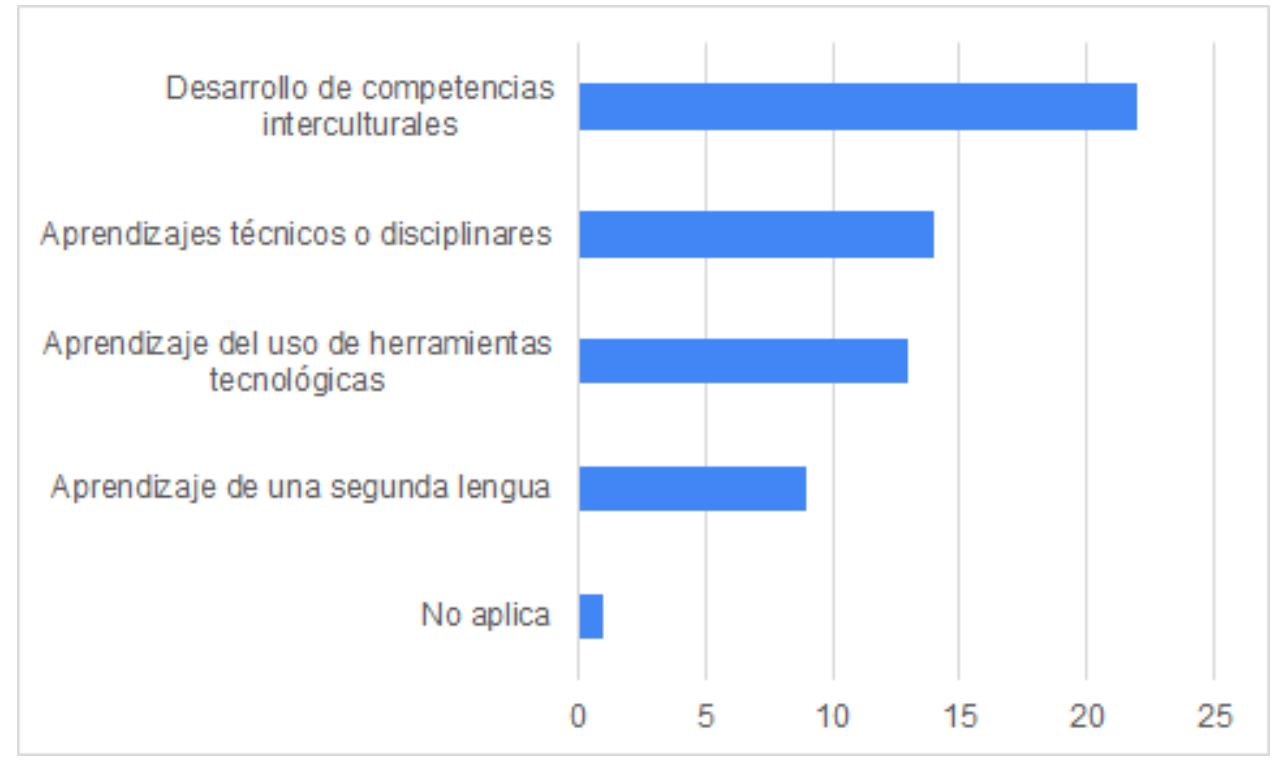

Figura 8. Aspectos que su IES tiene en cuenta para evaluar la experiencia en el intercambio virtual

Con respecto a la homologación de las experiencias de intercambio virtual (figura 9), 21 instituciones encuestadas contestaron que dichas actividades si se homologan, mientras que 3 informaron no efectuar homologaciones para el intercambio virtual y 2 contestaron no saber si esta estrategia se lleva a cabo en su IES.

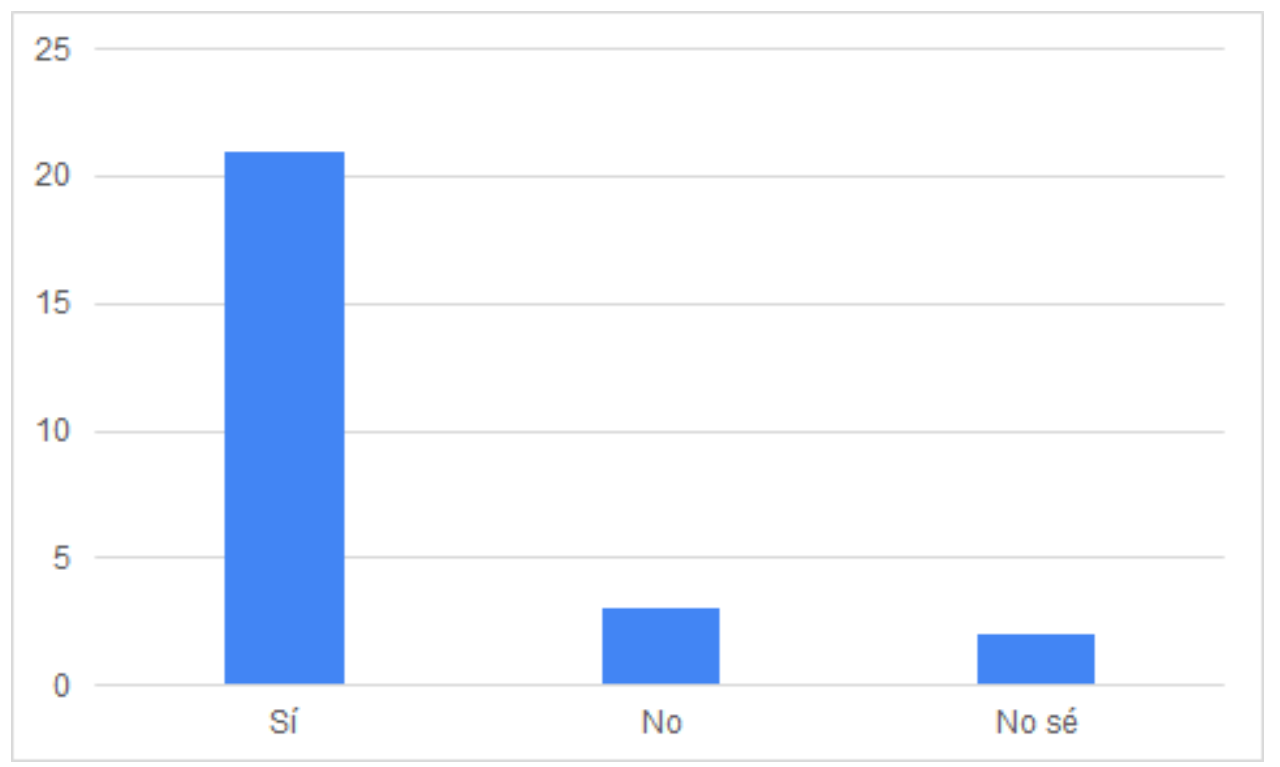

Figura 9. Homologación del intercambio virtual

Objetivo No 3. Relacionar áreas de conocimiento, niveles de formación y países socios con las que se realizan acciones de intercambio virtual estudiantil.

En la Figura 10 se encuentra que en general, todas las áreas del conocimiento indagadas, Ilevan a cabo la estrategia del intercambio virtual. Se observa que 24 IES reportan a las 
ciencias administrativas y contables, le siguen muy de cerca las ciencias sociales y humanidades con 23, las ingenierías con 16; las ciencias de la salud y educación, son identificadas por $11 \mathrm{IES}$, por último en nivel inferior las ciencias básicas 8, y arte y arquitectura señaladas por 6 IES.

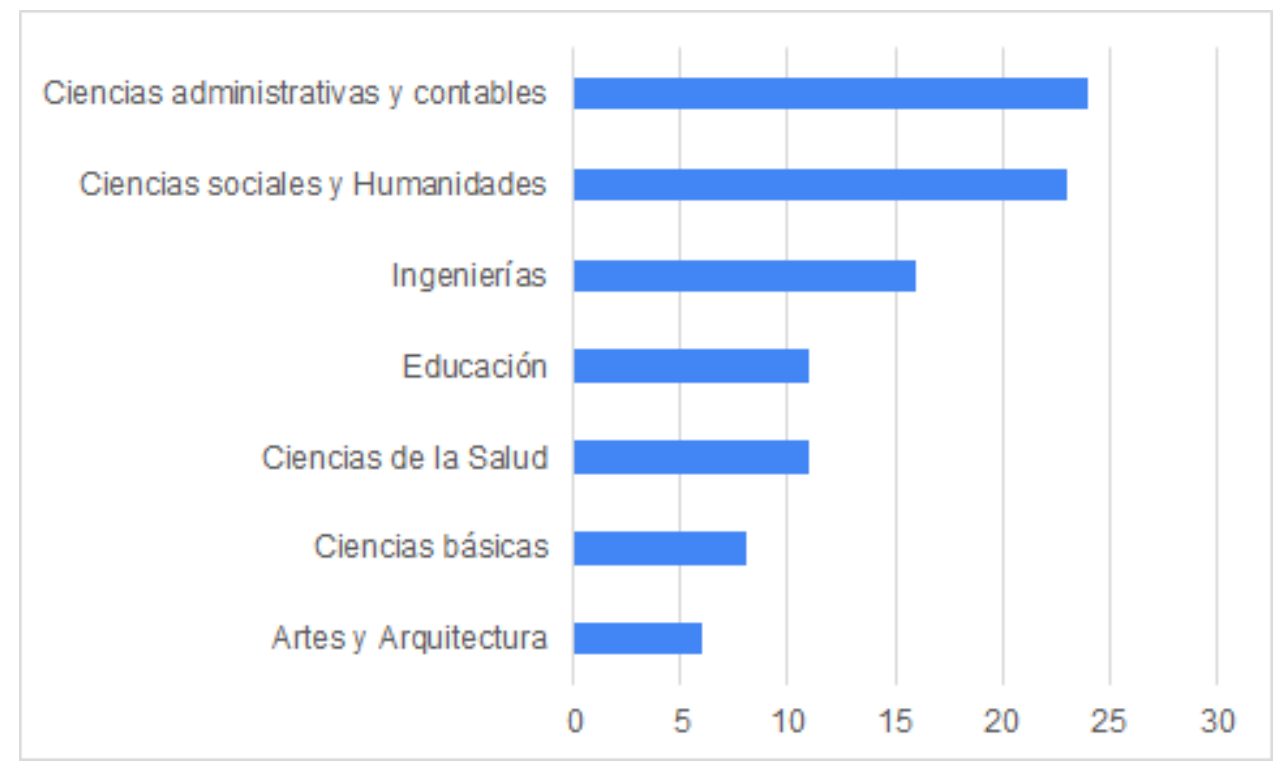

Figura 10. Áreas del conocimiento en las que realiza intercambio virtual

También se indagó sobre el nivel de formación en el que se encuentran los estudiantes que participan en intercambios virtuales. Los tres niveles indagados fueron tecnológico, pregrado y posgrado; destacándose pregrado por 24, posteriormente el nivel tecnológico con una representación de 7 y en menor grado posgrados es escogido por 3 IES, como se observa en la Figura 11:

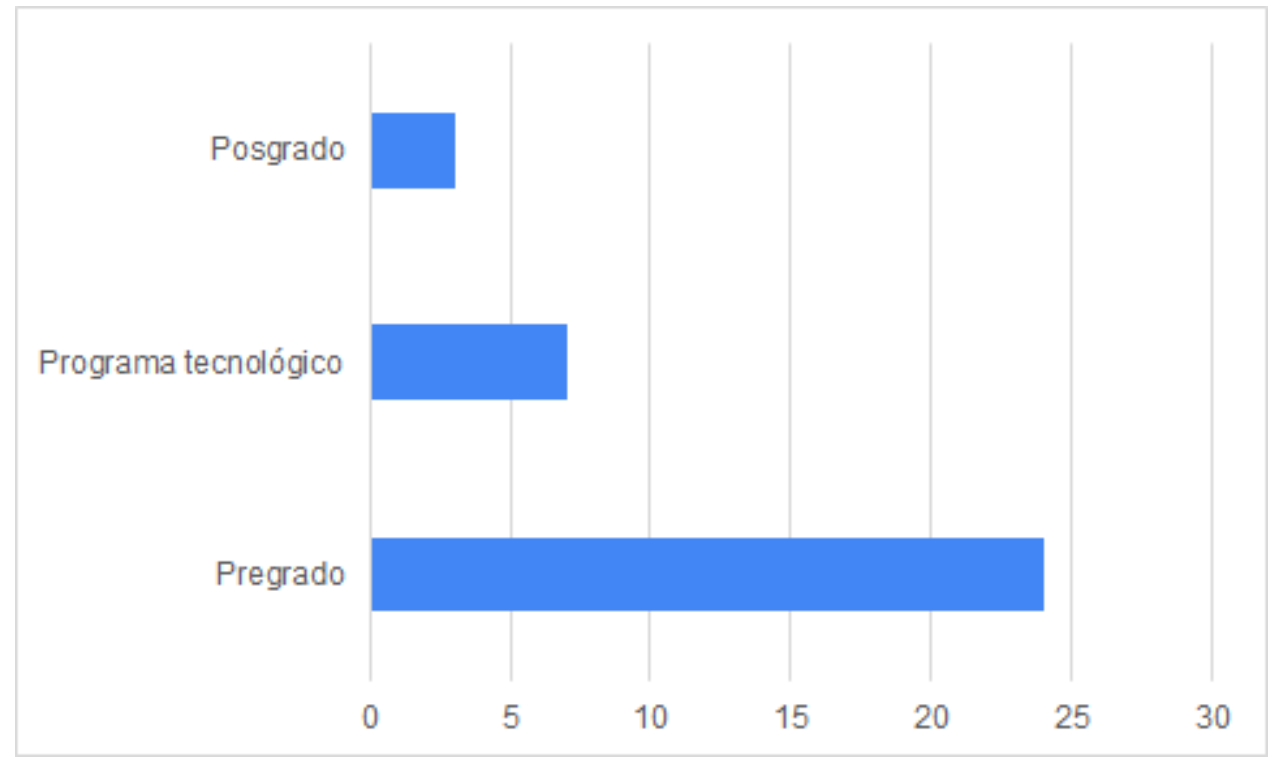

Figura 11. Nivel de formación de participantes en intercambio virtual 
Se indagó sobre los países socios con los que las instituciones realizan intercambio virtual estudiantil. La Figura 12. señala que fueron identificados 17 países con los que las IES realizan intercambios virtuales. Se observa que (24) IES, respondieron que México es el país más representativo, con un 92.3\%, le siguen Argentina con un 65.38\%, Perú 50\% y Chile $38.46 \%$. En menor medida se realizan intercambios virtuales con: España, Brasil, Ecuador, Estados Unidos, Uruguay, Colombia, Alemania, República Dominicana, Francia; los tres con menor porcentaje son Canadá, Panamá y Bélgica, con un 3.85\% cada uno.

También se evidencia que los primeros cuatro países elegidos por las IES del Caribe colombiano, son países de América Latina. Es importante destacar que el $64.70 \%$ corresponde a países hispanoparlantes; los otros idiomas representados son portugués, inglés, alemán, y francés; esto también expone un desafío para impulsar el dominio de otros idiomas en estudiantes y docentes.

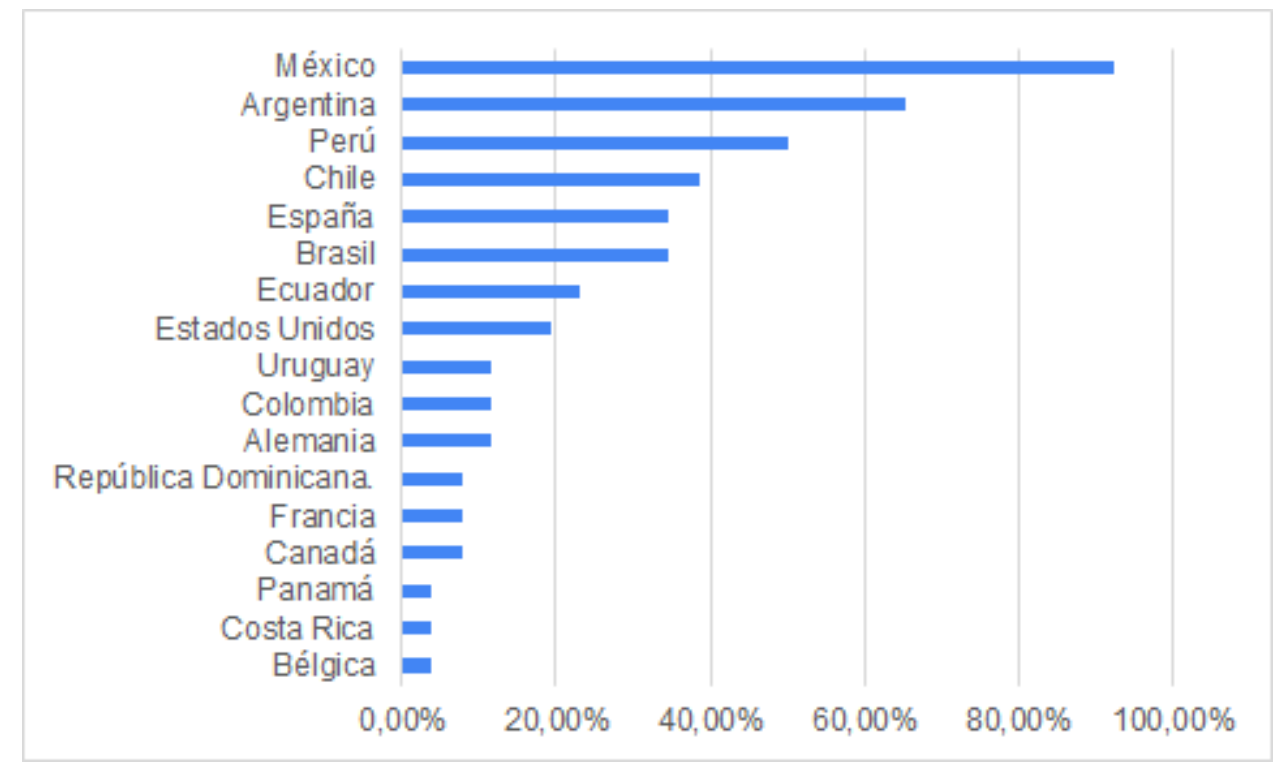

Figura 12. Países con los que su institución realiza iniciativas de intercambio virtual

Se indaga a las IES participantes, los tipos de entidades con las que se realizan los intercambios virtuales estudiantiles. La totalidad de las IES respondieron que los realizan con Instituciones de Educación Superior, 7 incluyen las ONG, y en menor nivel empresas con 4; para asociaciones como la UDUAL y entidades de gobierno, cada una fue referenciada por 2 IES (ver Figura 13): 


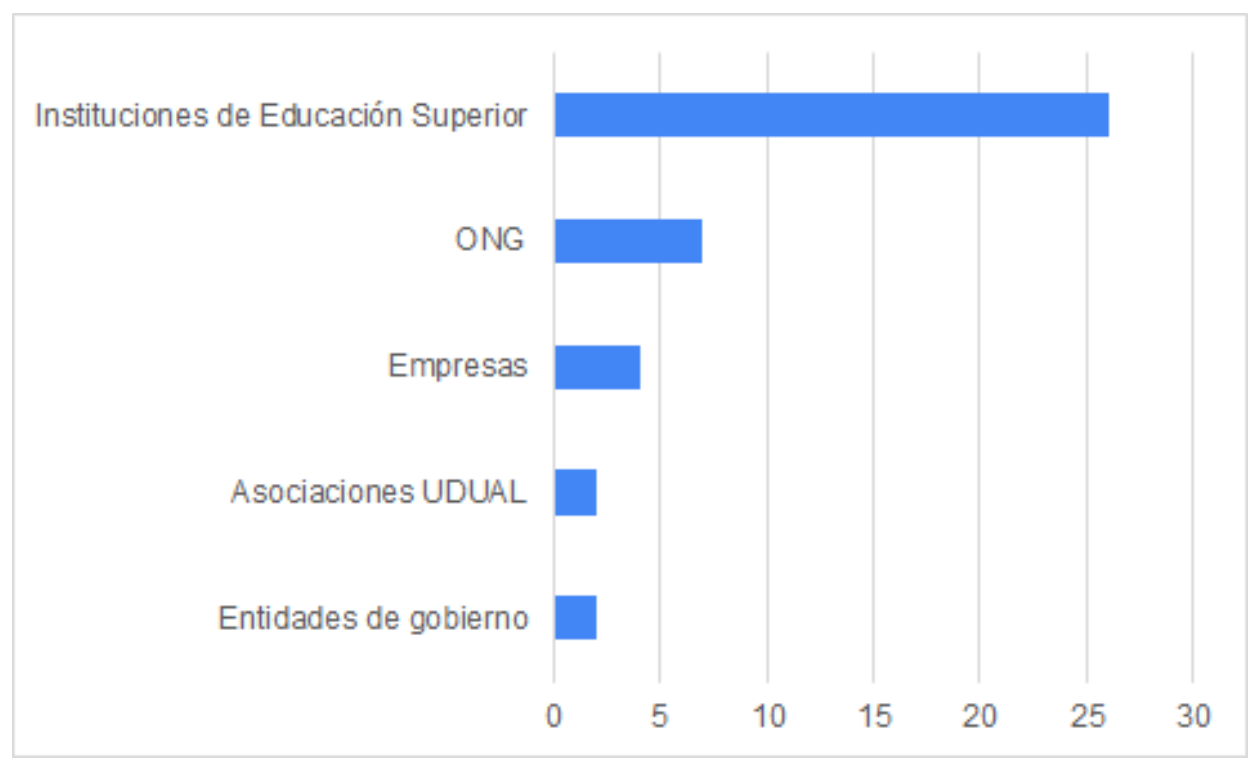

Figura 13. Tipo de entidades con los que realiza intercambios virtuales

Seguidamente en la Figura 14, se observa el resultado de la indagación acerca del marco de cooperación para los esquemas de colaboración internacional. La pregunta permitía escoger tres o más opciones. 24 IES manifestaron realizar el intercambio virtual mediante los convenios con otras instituciones, seguido del intercambio virtual en redes escogido por 21, y 11 en el marco de asociaciones. En este sentido, es evidente que las IES reconocen en los convenios, la principal vía para desarrollar intercambio virtual.

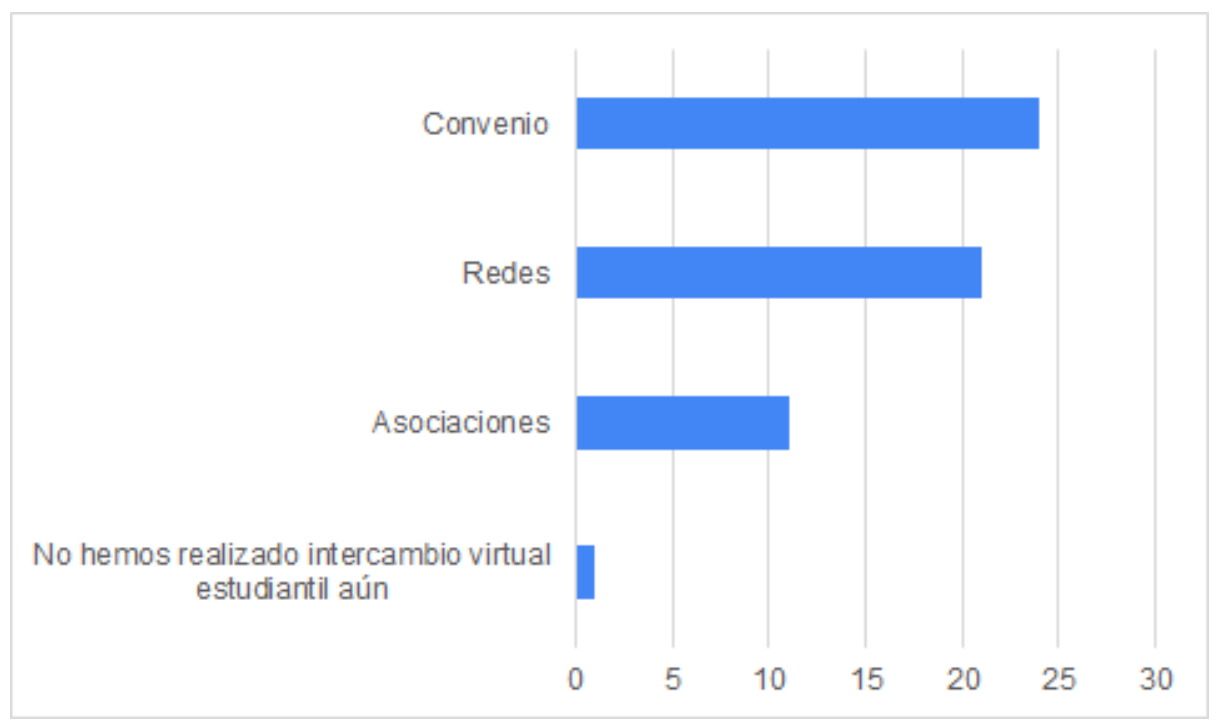

Figura 14. Esquema de colaboración internacional mediante el cual su institución realiza intercambios virtuales 


\section{Objetivo 4. Identificar desafíos en la implementación de esquemas de intercambios virtuales estudiantiles.}

Los desafíos que identifican las IES para la implementación de intercambios virtuales radican en las capacidades institucionales, en lo que concierne a infraestructura tecnológica una buena conexión a internet y acceso a equipos para la comunidad estudiantil. Asimismo, la ausencia de capacitación docente para el manejo adecuado de las plataformas virtuales y en pedagogía para el desarrollo de clases y motivación del docente para la gestión del intercambio virtual. También, la ausencia de capacitación estudiantil en el uso de TIC y su motivación para la participación en el intercambio virtual.

En cuanto a aspectos administrativos señalaron la medición del impacto en los resultados de aprendizaje del estudiante, la continuidad de la estrategia en la vuelta a la presencialidad, el desarrollo de intercambio virtual en asignaturas prácticas como laboratorios y temas de salud, la diferencia de calendarios de las instituciones pares de cara a coordinar las sesiones de intercambio virtual y la virtualización de los procesos de intercambio, teniendo en cuenta que previamente eran presenciales, como ilustra la Figura 15:

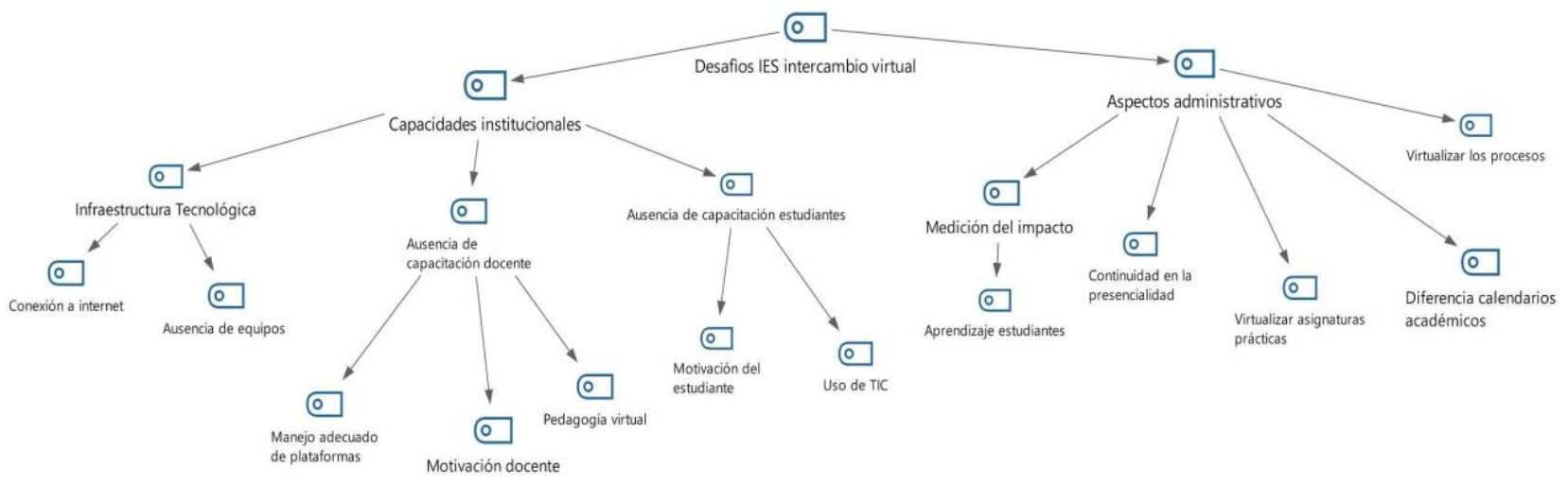

Figura 15. Desafíos del intercambio virtual estudiantil

La Figura 16 nos indica que 21 instituciones, manifestaron su intención de continuar con acciones de intercambio virtual en el futuro, 4 aún no lo saben y sólo 1 IES considera que no continuará aplicando la estrategia. 


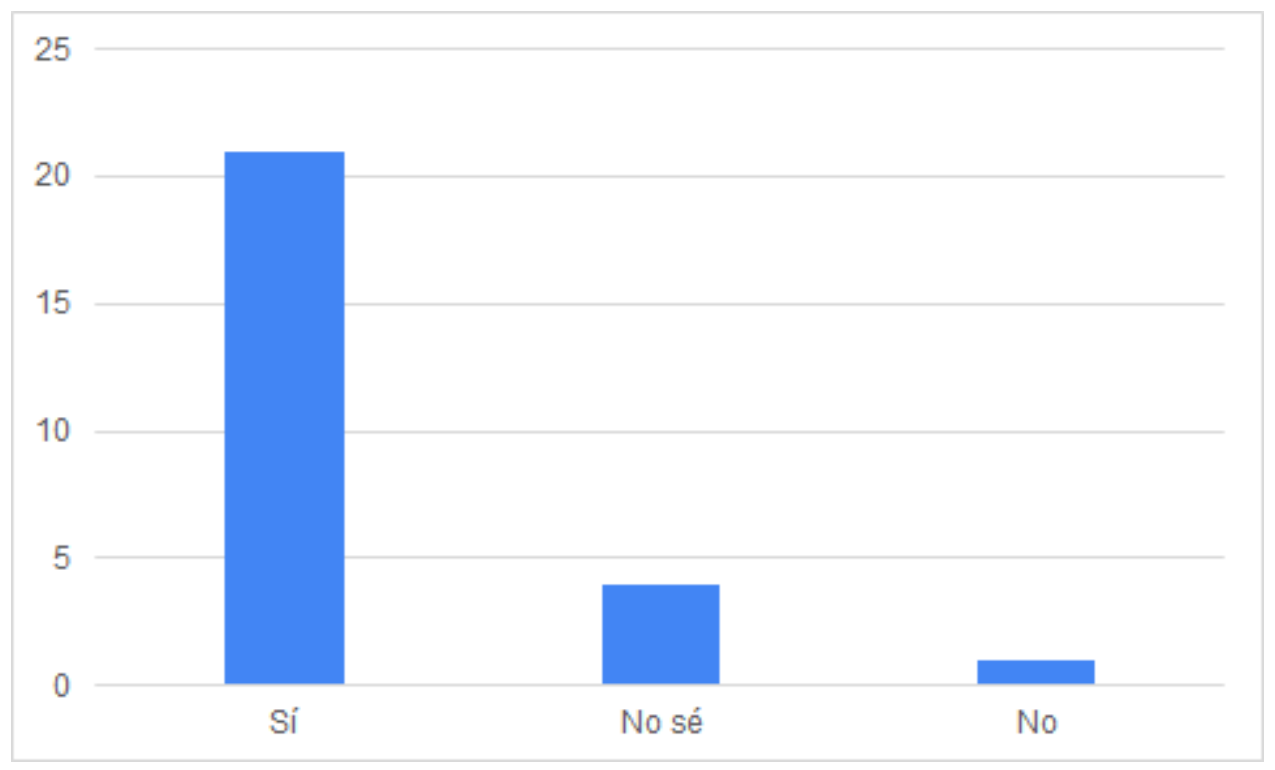

Figura 16. Continuará su IES con intercambios virtuales en el futuro

Se recogieron igualmente algunas recomendaciones, por medio de una pregunta abierta, de cara a una mejor preparación por parte de las IES para desarrollar intercambio virtual. Es imprescindible contar con infraestructura tecnológica en lo que concierne a equipos, plataforma y conexión a internet. Se deben implementar planes de formación dirigido a docentes en temas de aprendizaje colaborativo internacional (COIL), uso de TIC, mediación pedagógica y trabajo en aulas internacionalizadas $y$, a estudiantes para que usen apropiadamente las TIC. Asimismo, se requiere el diseño de un manual para el desarrollo de intercambio virtual que cuente con la validación de las autoridades institucionales. De la mano de esta normativa se requiere el desarrollo de planeación estratégica que contemple la evaluación de la experiencia estudiantil, el impacto de las acciones de intercambio virtual y el mejoramiento continuo.

En cuanto a los procesos administrativos se resalta la necesidad de flexibilizar el currículo para la ampliación y mejora de la oferta de intercambios virtuales e incorporar las TIC para la replicabilidad de la estrategia. Asimismo, definir a nivel institucional las asignaturas a ofertar previo al lanzamiento de la convocatoria de intercambio virtual para evitar contratiempos.

Por último, resalta la importancia del trabajo en red con otras IES para avanzar en esquemas de intercambio virtual, compartir capacidades, buenas prácticas y gestionar nuevos convenios. Asimismo, trabajar de la mano con el gobierno para solicitar un reconocimiento formal de las acciones de intercambio virtual como indicadores de internacionalización, dentro de los esquemas actuales de aseguramiento de la calidad en Colombia, como se observa en la Figura 17: 


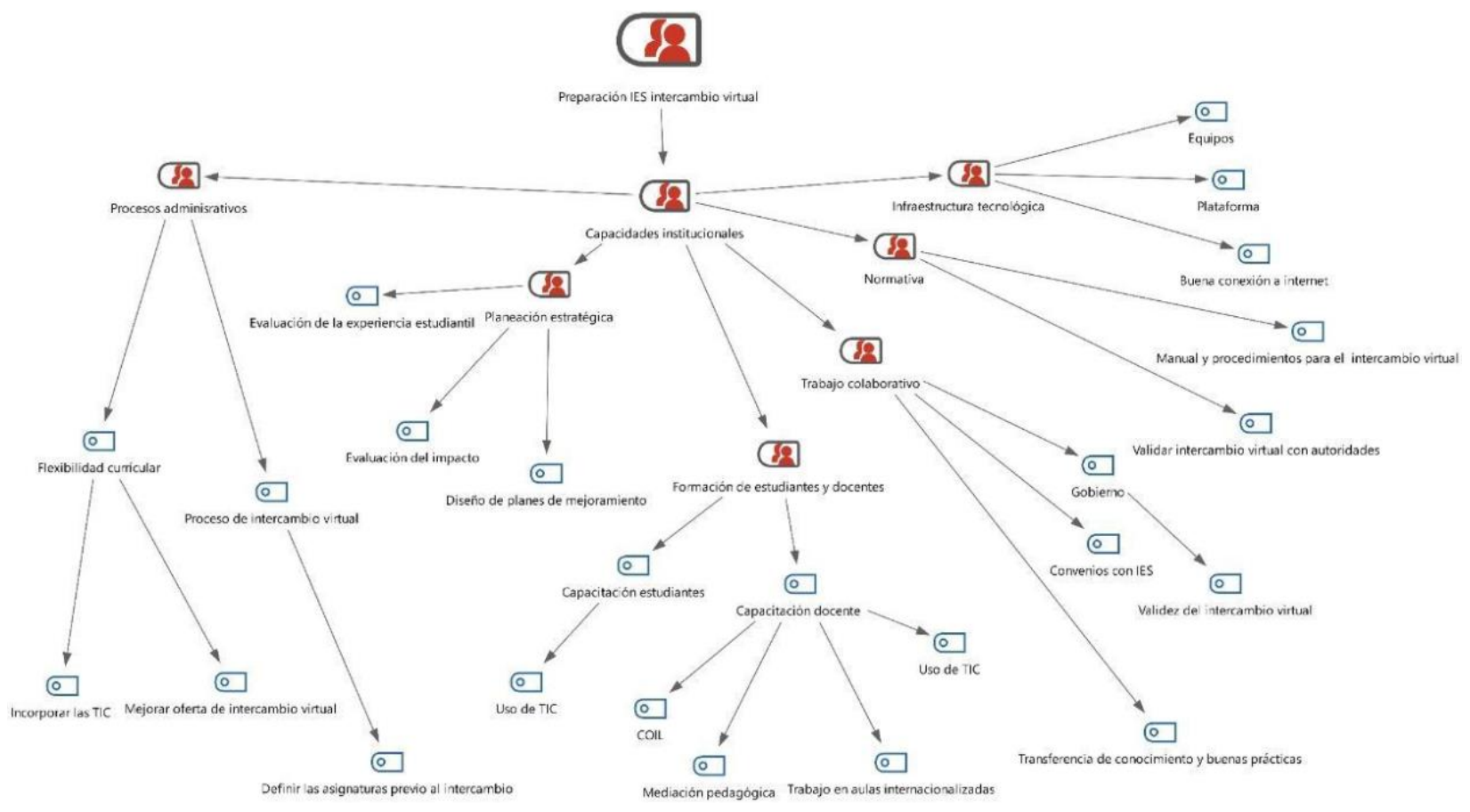

Figura 17. Preparación de IES para intercambios virtuales

\section{DISCUSIÓN Y CONCLUSIONES}

Los hallazgos evidencian que las instituciones del Nodo Caribe en respuesta a la pandemia causada por el Covid-19 acentuaron el desarrollo del intercambio virtual estudiantil como medio para fortalecer su estrategia de internacionalización en casa.

Se observa una tendencia interesante hacia el desarrollo de actividades como pasantías de investigación virtual y prácticas empresariales que inciden positivamente en el perfil global de los estudiantes. No obstante, actividades como COIL y clases espejo que requieren de gestión docente son efectuadas en menor medida, en los hallazgos del estudio se destaca que uno de los desafíos señalados por los participantes fue la necesidad de sensibilizar al docente de cara a la continuidad de la iniciativa. Por su parte, la actividad más frecuente, el intercambio académico virtual se encuentra ligado al currículo, por lo cual debería contar con un proceso de evaluación y homologación correspondiente.

En cuanto a los socios para el desarrollo de intercambio virtual estudiantil, es importante destacar la participación de algunas instituciones encuestadas en los esquemas de intercambio virtual promovidos por la Organización Universitaria Interamericana y la Asociación Colombiana de Universidades que trabajan con instituciones de Latino América, retomando a Schenker (2013) el trabajo en red incluye a los estudiantes en comunidades de aprendizaje virtual y los motiva a conocer otras culturas. Conviene resaltar la importancia de fortalecer esta estrategia con países de lengua materna distinta al español. 
Las disciplinas que más reportaron el desarrollo de intercambio virtual son las ciencias blandas como las administrativas y contables, las ciencias sociales y humanidades, lo cual coincide con el estudio de (Jager et al., 2019).

En cuanto a las prácticas de evaluación se resalta la medición de resultados de aprendizaje valorando aspectos cualitativos como la interacción con otras culturas y el aprendizaje multicultural, acorde con lo expuesto por Tiven et al. (2018) y el uso de métodos directos como informe, evaluación por proyecto, examen, rúbrica (Deardoff, 2015).

Por otro lado, la evaluación de la satisfacción estudiantil se realiza mediante métodos indirectos: encuestas, entrevistas e informes que dan cuenta de la percepción del estudiante en torno al aprendizaje (Deardoff, 2015). El método más frecuente fue la aplicación de encuestas resaltadas por los autores Guadamillas Gómez (2017) como método efectivo para evaluar la satisfacción de los estudiantes frente a la ejecución y resultado del intercambio virtual.

Las instituciones dan prioridad a las competencias interculturales como el aspecto más frecuente para evaluar la experiencia de intercambio virtual (Guth et al. 2014). Sin embargo, todavía hay dudas frente al desarrollo de estas competencias por medio de la virtualidad exclusivamente, en línea con lo expuesto por Rienties et al. (2020).

En cuanto a los desafíos se destaca la infraestructura tecnológica una buena conexión a internet y acceso a equipos para la comunidad estudiantil, aspectos señalados por Cavalari (2019). Asimismo, la necesidad de formación del docente como facilitador de los intercambios virtuales y del diseño del currículo tal como lo exponen Cavalari y Aranha (2019). La motivación del docente es otro aspecto clave para la continuidad de la estrategia, por lo cual se recomienda el aporte de incentivos y el reconocimiento del tiempo de preparación de las sesiones en la carga horaria, tal como lo señala The Evaluate Group (2019).

En cuanto a aspectos administrativos nuevamente se resalta la medición del impacto en los resultados de aprendizaje del estudiante. La continuidad de la estrategia en la vuelta a la presencialidad, por lo cual se recomienda la inclusión de una estrategia de movilidad virtual en la política institucional para que cuente con un aval institucional tal como lo exponen Garcés y O'Dowd (2020). La diferencia de calendarios de las instituciones pares de cara a coordinar las sesiones de intercambio virtual, aspecto que destaca Cavalari (2019) y la virtualización de los procesos de intercambio tanto en asignaturas tradicionales y las de enfoque práctico, teniendo en cuenta que previamente eran presenciales.

De cara a la continuidad de la estrategia de intercambio virtual en las Instituciones del Caribe, se evidencia que es clave el fortalecimiento del proceso de gestión, en lo que puede aportar la confección de guías pertinentes al contexto institucional, en las que se establezcan pasos, criterios, responsables y referentes estratégicos para la sensibilización de la comunidad. Asimismo, el desarrollo de capacitación docente a nivel pedagógico y en manejo de las TIC para el diseño de los intercambios virtuales, resultados de aprendizaje virtual e innovación en metodologías de aprendizaje, reivindicando el rol docente como guía y facilitador según lo expuesto por Moreira-Segura y Delgadillo-Espinoza (2015). Se hace indispensable desarrollar la infraestructura de la conectividad. 
Sobre las implicaciones prácticas de esta investigación, se puede indicar que sirve como guía a instituciones, especialmente aquellas ubicadas en el contexto latinoamericano, que desean empezar a implementar iniciativas de intercambio virtual, al facilitar información sobre los diversos tipos de intercambio virtual, las formas de evaluar y los desafíos para su implementación, así como se presentan aspectos administrativos y logísticos clave para preparar a una institución interesada en implementar esquemas de intercambio virtual. Sobre las limitaciones de este estudio se puede exponer que está limitado a un grupo de instituciones (26), por lo cual sería relevante realizar mediciones a un número mayor de instituciones; asimismo, merece la pena realizar estudios similares en diversas partes de Latinoamérica y el mundo, con el fin de evidenciar las tendencias y enfoques desde cada una de las latitudes. También se requieren estudios que profundicen sobre métodos para evaluar las estrategias de intercambio virtual.

\section{REFERENCIAS}

Association of American Colleges and Universities (AAC\&U). (2009). Inquiry and analysis VALUE rubric. https://cutt.ly/ZjxLaW7

Behar, D. (2008). Metodología de la investigación. Editorial Shalom.

Cavalari, S. M. S. (2019). Integrating telecollaborative language learning into Higher Education: a study on teletandem practice. BELT - Brazilian English Language Teaching Journal, 9(2), 417-432. https://doi.org/10.15448/2178-3640.2018.2.31927

Cavalari, S. y Aranha, S. (2019). The Teacher's Role in Telecollaborative Language Learning: The Case of Institutional Integrated Teletandem. Revista Brasileira de Linguística Aplicada, 19(3), 555-578. https://doi.org/10.1590/1984-6398201913576

Congreso de Colombia (1992). Ley 30 de 1992, del 28 de diciembre de 1992, por la cual se organiza el servicio público de la Educación Superior. Diario Oficial No. 40.700 del 29 de diciembre de 1992.

Deardoff D.K. (2015). Outcomes assessment for international educators. Stylus Publishing.

Camacho Lizárraga, M. (2017). Políticas institucionales y exclusión en la movilidad estudiantil internacional. Casos en México. Universidades, 74, 63-73.https://cutt.ly/6gfBQRA

Galván Acosta, F. y Moreno Correa, J. (2017). Intercambio virtual entre estudiantes de psicología en Latinoamérica. Integración Académica en Psicología, 5(15), 42-54. https://cutt.ly/Uf1IK2L

Garcés, P. y O'Dowd, R. (2020). Upscaling Virtual Exchange in University Education: Moving From Innovative Classroom Practice to Regional Governmental Policy. Journal of Studies in International Education, 1-18. https://doi.org/10.1177/1028315320932323

Guadamillas Gómez, M. (2017). Los intercambios virtuales lingüísticos y culturales en educación superior: un estudio de caso. Apertura -Universidad de Guadalajara-, 9(1), 8-21. https://doi.org/10.32870/ap.v9n1.1016

Guth, S., Helm, F. y O'Dowd, R. (2014) Telecollaborative foreign language networks in European universities: A report on current attitudes and practices. Bellaterra Journal of Teaching and Learning Language \& Literature, 7(4), 1-14. https://cutt.ly/rf10QGy 
Jager S., Nissen E., Helm F., Baroni A. y Rousset I. (2019). Virtual Exchange as Innovative Practice across Europe Awareness and Use in Higher Education. https://bit.ly/30PXG4L

Moreira-Segura, C., y Delgadillo-Espinoza, B. (2015). La virtualidad en los procesos educativos: reflexiones teóricas sobre su implementación. Revista Tecnología En Marcha, 28(1), 121-129. https://doi.org/10.18845/tm.v28i1.2196

Niño, V. (2011). Metodología de la Investigación. Ediciones de la U.

O'Dowd, R (2018). From telecollaboration to virtual exchange: state-of-the-art and the role of UNICollaboration in moving forward. Journal of Virtual Exchange, 1, 1-23. https://doi.org/10.14705/rpnet.2018.jve.1

Rienties B., Lewis T., O'Dowd R., Rets I. y Rogaten J. (2020). The impact of virtual exchange on TPACK and foreign language competence: reviewing a large-scale implementation across 23 virtual exchanges. Computer assisted language learning, 1-27. doi: 10.1080/09588221.2020.1737546

Schenker, T. (2013). The Effects of a Virtual Exchange on Students' Interest in Learning About Culture. Foreign Language Annals, 46(3), 491-507. https://doi.org/10.1111/flan.12041

Stevens Initiative (2019). Virtual Exchange Impact and Learning Report. https://cutt.ly/Gf1IB7j

Telles, João A. (2015). Learning foreign languages in teletandem: Resources and strategies. DELTA: Documentação de Estudos em Lingüística Teórica e Aplicada, 31(3), 603632. https://doi.org/10.1590/0102-4450226475643730772

The Collaboratory (2019). Virtual Exchange Toolkit. Bureau of Educational and Cultural Affairs US Department of State. https://cutt.ly/bjxL6AC

The Evaluate Group. (2019). Evaluating the impact of virtual exchange on initial teacher education: a European policy experiment. Research-publishing.net. https://doi.org/10.14705/rpnet.2019.29.9782490057337

Tiven, M. B., Fuchs, E. R., Bazari, A., y MacQuarrie, A. (2018). Evaluating Global Digital Education: Student Outcomes Framework. Bloomberg Philanthropies and the Organisation for Economic Co-operation and Development. https://cutt.ly/gjxKU9p

Verzella, M. (2018). Virtual Exchange between Cross-Cultural Teams: A Sustainable Path to the Internationalization of College Courses. Transformative Dialogues: Teaching \& Learning Journal, 11(13), 1-13. https://cutt.ly/GgfBIIf

\section{Para citar este artículo:}

Lafont Castillo, T. I., Echeverría King, L. F., y Álvarez Ruíz, L. P. (2021). El intercambio virtual: experiencias desde instituciones en el Caribe colombiano. Edutec. Revista Electrónica De Tecnología Educativa, (75), 90-109. https://doi.org/10.21556/edutec.2021.75.1893 